\section{Growth and Nutrient Partitioning of 'TifEagle' Bermudagrass as Influenced by Nitrogen and Trinexapac-ethyl}

\author{
Patrick E. McCullough \\ Department of Plant Biology and Pathology, Rutgers, The State University of \\ New Jersey, New Brunswick, NJ 08901-8520
}

Haibo Liu, ${ }^{2}$ Lambert B. McCarty, ${ }^{3}$ and Ted Whitwell ${ }^{3}$

Department of Horticulture, Clemson University, Clemson, SC 29634-0375

Joe E. Toler
Department of Applied Economics and Statistics, Clemson University, Clemson,
SC 29634-0319

Additional index words. plant growth regulator, PGR, dwarf bermudagrass

\begin{abstract}
Dwarf-type bermudagrasses [Cynodon dactylon (L.) Pers. $\times$ C. transvaalensis Burtt-Davey] tolerate long-term golf green mowing heights but require heavy nitrogen $(N)$ fertilizations. Inhibiting leaf growth with trinexapac-ethyl (TE) could reduce shoot growth competition for root reserves and improve nutrient use efficiency. Two greenhouse experiments evaluated four N levels, 6 (N6), 12 (N12), 18 (N18), and 24 (N24) kg N/ha/week, with TE at 0 and $0.05 \mathrm{~kg}^{\circ} \mathrm{ha}^{-1}$ a.i. every 3 weeks to assess rooting, nutrient allocation, clipping yield, and chlorophyll concentration of 'TifEagle' bermudagrass grown in PVC containers built to U.S. Golf Association specification. Trinexapac-ethyl enhanced turf quality on every date after initial application. After 8 weeks, high $\mathrm{N}$ rates caused turf quality decline; however, TE treated turf averaged about $25 \%$ higher visual quality from nontreated turf, masking quality decline of high $\mathrm{N}$ fertility. 'TifEagle' bermudagrass treated with TE had clippings reduced $52 \%$ to $61 \%$ from non-TE treated. After 16 weeks, bermudagrass treated with TE over all $\mathrm{N}$ levels had $43 \%$ greater root mass and $23 \%$ enhanced root length. Compared to non-TE treated turf, leaf $N$, $P$, and $K$ concentrations were consistently lower in TE treated turf while $\mathrm{Ca}$ and $\mathrm{Mg}$ concentrations were increased. Root $\mathrm{N}$ concentrations in TE treated turf were $8 \%$ to $11 \%$ higher for $\mathrm{N12}, \mathrm{N} 18$, and $\mathrm{N} 24$ fertilized turf than respective $\mathrm{N}$ rates without TE. Compared to non-TE treated turf, clipping nutrient recoveries were reduced $69 \%$ to $79 \%$ by TE with $25 \%$ to $105 \%$ greater nutrients recovered in roots. Bermudagrass treated with TE had higher total chlorophyll concentrations after 8 and 12 weeks. Overall, inhibiting 'TifEagle' bermudagrass leaf growth appears to reallocate nutrients to belowground tissues, thus improving nutrient use efficiency and root growth. Chemical name used: trinexapac-ethyl, [4-(cyclopropyl-[ $\alpha]$-hydroxymethylene)-3,5-dioxo-cyclohexane carboxylic acid ethylester].
\end{abstract}

Hybrid bermudagrass is the most commonly used warm-season turfgrass for putting greens in the warm-humid climatic regions. Bermudagrass putting greens are the most heavily fertilized turfgrasses due to annual nitrogen $(\mathrm{N})$ requirements ranging from 390 to $1,175 \mathrm{~kg} \cdot \mathrm{ha}^{-1}$ to meet growth requirements and compensate for nutrient loss through daily clipping removal (McCarty and Miller, 2002). Dwarf bermudagrass cultivars tolerate routine close mowing heights and provide southern golf courses putting green quality comparable to creeping bentgrass (Agrostis palustris Huds.) (Hanna and Elsner, 1999; McCarty and Miller, 2002). However, the close mowing of cultivars, such as 'TifEagle', has apparently restricted root systems compared to traditional putting

Received for publication 5 Dec. 2004. Accepted for publication 25 Mar. 2005. Contribution from the South Carolina Agricultural Experiment Station. ${ }^{1}$ Program associate.

${ }^{2}$ Associate professor and corresponding author; e-mail haibol@clemson.edu.

${ }^{3}$ Professor. green cultivars like 'Tifdwarf' bermudagrass (White, 1998).

Promoting root growth of dwarfbermudagrass may present challenges for successful long term culture. Low cutting height and frequent mowing are directly correlated with root growth restrictions of putting green turf(Fagerness and Yelverton, 2001; Liu and Huang, 2002). With potentially reduced photosynthetic capacity from finer leaves, dwarf-type bermudagrasses maintained at mowing heights as close as 3 $\mathrm{mm}$ may have a depletion of plant reserves available for root growth. Furthermore, high N requirements of bermudagrass putting greens may further exacerbate rooting problems of dwarf cultivars since shoots have priority for stored nutrients in belowground tissues.

Application of a plant growth regulator (PGR), trinexapac-ethyl (TE), to 'Tifway' bermudagrass has shown to reduce $\mathrm{N}$ allocation to leaf tissue while increasing $\mathrm{N}$ retention in rhizomes and roots (Fagerness et al., 2004). These findings may be of greater importance to dwarf-type cultivars due to higher fertility requirements for bermudagrass putting green maintenance. Greater nutrients stored in belowground tissues following TE applications may be readily allocated to prevent leafdeficiencies, thus improving nutrient use efficiency of heavily fertilized dwarf bermudagrass greens.

Trinexapac-ethyl, a cyclohexadione gibberellic acid (GA) inhibitor, interferes with GA biosynthesis by blocking $3 \beta$-hydroxylase conversion of GA to $_{20}$ (Rademacher, 2000). Sequential applications of TE on 'Tifway' bermudagrass provide consistent growth suppression, avoidance of post inhibition growth enhancement, improvements in turf color, and delay fall dormancy (Johnson, 1997; Fagerness and Yelverton, 2000; Richardson, 2002). Trinexapac-ethyl also improves turf grown under stressful conditions that may restrict root growth. 'Diamond' zoysiagrass (Zoysia matrella (L.) Merr) treated with TE at 0.048 $\mathrm{kg} \cdot \mathrm{ha}^{-1}$ monthly and $0.096 \mathrm{~kg} \cdot \mathrm{ha}^{-1}$ bimonthly under reduced light conditions displayed higher root mass, higher root viability, and improved photosynthesis (Qian and Engelke, 1999). Applications of TE at 0.042 and $0.070 \mathrm{~kg} \cdot \mathrm{ha}^{-1}$ on creeping bentgrass greens under $80 \%$ shade have shown to increase turf cover, tillering, and fructose content but had no effect on root mass compared to untreated turf (Goss et al., 2002). In a 2-year study, monthly applications of TE at $0.05 \mathrm{~kg} \cdot \mathrm{ha}^{-1}$ did not affect rooting of a 'Penncross' creeping bentgrass golf green (Fagerness and Yelverton, 2001).

Since TE is an effective PGR for higher mowed bermudagrasses and creeping bentgrass putting greens, research is warranted on physiological responses of dwarf bermudagrass putting greens to TE applications. Although turf color and quality enhancements have been documented from TE applications, effects on primary, secondary, and micronutrient partitioning are limited. The objectives of this experiment were to investigate effects of four weekly $\mathrm{N}$ inputs with TE on nutrient allocation, clipping yield, rooting, and chlorophyll concentrations of 'TifEagle' bermudagrass.

\section{Materials and Methods}

Two studies were conducted at the Clemson University Greenhouse Research Complex, Clemson, SC from September 2002 to January 2003 (Study 1) and January to May 2003 (Study 2). Greenhouse day/night temperatures were about $24 / 19^{\circ} \mathrm{C}$. The experimental design was a randomized complete block with four replication of eight experimental units per block. To minimize local environmental variations, blocks were rotated weekly and experimental units re-randomized within. Supplemental lighting was added at approximately $50 \mu \mathrm{mol} \cdot \mathrm{m}^{-2} \cdot \mathrm{s}^{-1}$ for about $3 \mathrm{~h} \cdot \mathrm{d}^{-1}$ to compensate for reduced natural lighting during winter months.

Sod was collected from a 'TifEagle' bermudagrass green established in July of 2002 at the Turf Service Center, Clemson, S.C. Soil was washed and plugs placed in polyvinyl chloride containers with $40 \mathrm{~cm}$ depths and $324 \mathrm{~cm}^{2}$ surface areas built to U.S. Golf Association specification (U.S. Golf Association Green Section Staff, 1993) with an 85 sand : 15 peatmoss (by volume) rootzone mix. Start- 
ing fertilizer (9N-8P-14K) at $48 \mathrm{~kg} \mathrm{~N} /$ hawas mixed into the soil, and bermudagrass was established 4 and 6 weeks in Studies 1 and 2 , respectively, before treatment initiation. Urea-based $\mathrm{N}$ fertilizer $(16 \mathrm{~N}-2 \mathrm{P}-7 \mathrm{~K})$ with micronutrients was applied to all containers at $24 \mathrm{~kg} \mathrm{~N} / \mathrm{ha} 2$ weeks before initial treatments and at $12 \mathrm{~kg} \mathrm{~N} / \mathrm{ha} 8$ weeks after experiment initiation.

Bermudagrass was irrigated to field capacity and mowed daily with grass sheers (Black and Decker, Towson, Md.) at $4 \mathrm{~mm}$. The eight treatments were a factorial combination of four $\mathrm{N}$ levels and two TE levels. Ammonium nitrate $(34 \mathrm{~N}-0 \mathrm{P}-0 \mathrm{~K})$ solution was applied at $6,12,18$, and $24 \mathrm{~kg} \mathrm{~N} / \mathrm{ha} /$ week. Beginning 9 $\mathrm{d}$ after initial fertilizations, trinexapac-ethyl (1 EC) was applied at 0 or $0.05 \mathrm{~kg} \cdot \mathrm{ha}^{-1}$ every 3 weeks. Treatments were made at $300 \mathrm{~L} \cdot \mathrm{ha}^{-1}$ with a greenhouse spray chamber, Devries Manufacturing (Hollandale, Minn.) model SB6-094, with an air compressor supplying $170 \mathrm{kPa}$ pressure.

Turf quality was visually measured biweekly on a 1 to 9 scale, where 1 equaled dead turf and 9 equaled uniform, dark green turf. Clippings were harvested 16 weeks after initial treatment(WAIT) in Study 1 before roots were sampled to obtain nutrient recovery throughout the plant. Weekly clipping yield was introduced as a parameter in Study 2 to obtain $\mathrm{N}$ recovery in periodic intervals. Clippings were harvested and oven-dried at $80^{\circ} \mathrm{C}$ for $48 \mathrm{~h}$, then weighed. Roots, stolons, and rhizomes were harvested from entire lysimeters after 16 weeks, washed to remove soil and organic matter, dried, and weighed. Root length was determined by measuring the distance from where roots were no longer present in the soil profile to the top of the container.

Tissue and soil analyses were conducted at the Clemson Agriculture Service Laboratory. Nitrogen concentrations were determined us- ing a N combustion analyzer (FP528; LECO, Warrendale, Pa.). Other plant tissue nutrients were determined using wet ashing procedures with a block digester and an ICP autosampler (Digestion Block Magnum Series and model TJA-61E, respectively, Madison, Wis.). Soil nitrate-nitrogen extractions were made with an electrode (ISE, Beverly, Mass.). Chlorophyll concentrations were measured in $\mathrm{mg} \cdot \mathrm{g}^{-1}$ of fresh weight clippings, sampled 8 and 16 weeks after initial $\mathrm{N}$ treatments in Study 1 and 4, 8, 12 , and 16 weeks after initial $\mathrm{N}$ treatments for Study 2. Chlorophyll concentrations were measured with a spectrophotometer (Genesys 20 Thermo Spectronic model 4001/4, Waltham, Mass.) in $\mathrm{mg} \cdot \mathrm{g}^{-1}$ of fresh weight. Light absorbance was measured at $663 \mathrm{~nm}$ for chlorophyll a and $645 \mathrm{~nm}$ for chlorophyll b. Procedures for chlorophyll concentration analysis were similar to those described by Moran and Porath (1980). Data were subjected to analysis of variance with SAS General Linear Model procedure (SAS Institute, Cary, N.C.). Orthogonal polynomial contrasts examined linear and quadratic relationships between plant response and level of $\mathrm{N}$.

\section{Results}

Visual quality. Study $\times$ treatment interaction was not detected for visual quality, but date $x$ treatment interaction was detected. Therefore, results are presented by observation date for visual quality. Nitrogen $\times$ TE interactions were not significant on the first three dates but treatment interactions were significant on the last five dates (Table 1). Trinexapacethyl significantly enhanced turf quality on every date after week 2 . Turf quality linearly improved with increased $\mathrm{N}$ rate by week 4 . However, bermudagrass receiving 18 and 24 $\mathrm{kg} \mathrm{N} / \mathrm{ha} /$ week without TE reached their peak quality ratings by week 8 ; thereafter, quality declined. Conversely, turf receiving 18 and 24 $\mathrm{kg} \mathrm{N} / \mathrm{ha} /$ week with TE did not have quality decline, rather, quality was enhanced similar to bermudagrass receiving $12 \mathrm{~kg} \mathrm{~N} / \mathrm{ha} /$ week with TE. After week 6, quality enhancements from TE were most substantial when bermudagrass received $12 \mathrm{~kg} \mathrm{~N} / \mathrm{ha} /$ week or greater. From week 10 to 16, TE treated turf averaged about $25 \%$ higher visual quality from non-TE treated turf, apparently masking quality decline caused by higher $\mathrm{N}$ fertility.

'TifEagle' bermudagrass growth. Study $\times$ treatment interactions were not detected for clipping yield, roots, or stolon/rhizome mass. The $\mathrm{N} \times \mathrm{TE}$ interaction was significant for total clippings harvested. Bermudagrass receiving 12,18 , and $24 \mathrm{~kg} \mathrm{~N} /$ ha/week with TE had similar total clipping yield to non-TE treated turf receiving $6 \mathrm{~kg} \mathrm{~N} / \mathrm{ha} /$ week (Table 2 ). Compared to respective $\mathrm{N}$ rates, applications of TE reduced clippings by $52 \%, 61 \%, 56 \%$, and $61 \%$, for $6,12,18$, and $24 \mathrm{~kg} \mathrm{~N} / \mathrm{ha} /$ week, respectively. The $\mathrm{N} \times \mathrm{TE}$ interaction was not significant for root length, dry root mass, or thatch mass. 'TifEagle' bermudagrass treated with TE had $43 \%$ greater root mass than nonTE treated turf (Table 2). Increased $\mathrm{N}$ rate linearly and quadratically reduced root length and dry root mass, respectively. However, TE enhanced root length by $23 \%$ and bermudagrass receiving high $\mathrm{N}$ (18 and $24 \mathrm{~kg} \mathrm{~N} /$ ha/week) plus TE had root masses similar to low N (6 and $12 \mathrm{~kg} \mathrm{~N} /$ ha/week) without TE. Treatments did not affect stolon and rhizome mass after 16 weeks (data not shown).

Nutrient concentrations. Tissue test results were pooled from two studies due to a lack of meaningful study $\times$ treatment interactions. Sample $\times$ TE interactions were detected for leaf nutrient concentrations; therefore, results are presented by sampling date(Fig. 1). Sample $\times \mathrm{N}$ interactions were not detected for leaf nutrient concentrations so results were pooled over all

Table 1. Turf quality for 'TifEagle' bermudagrass treated with $\mathrm{N}$ and trinexapac-ethyl in two combined greenhouse experiments.

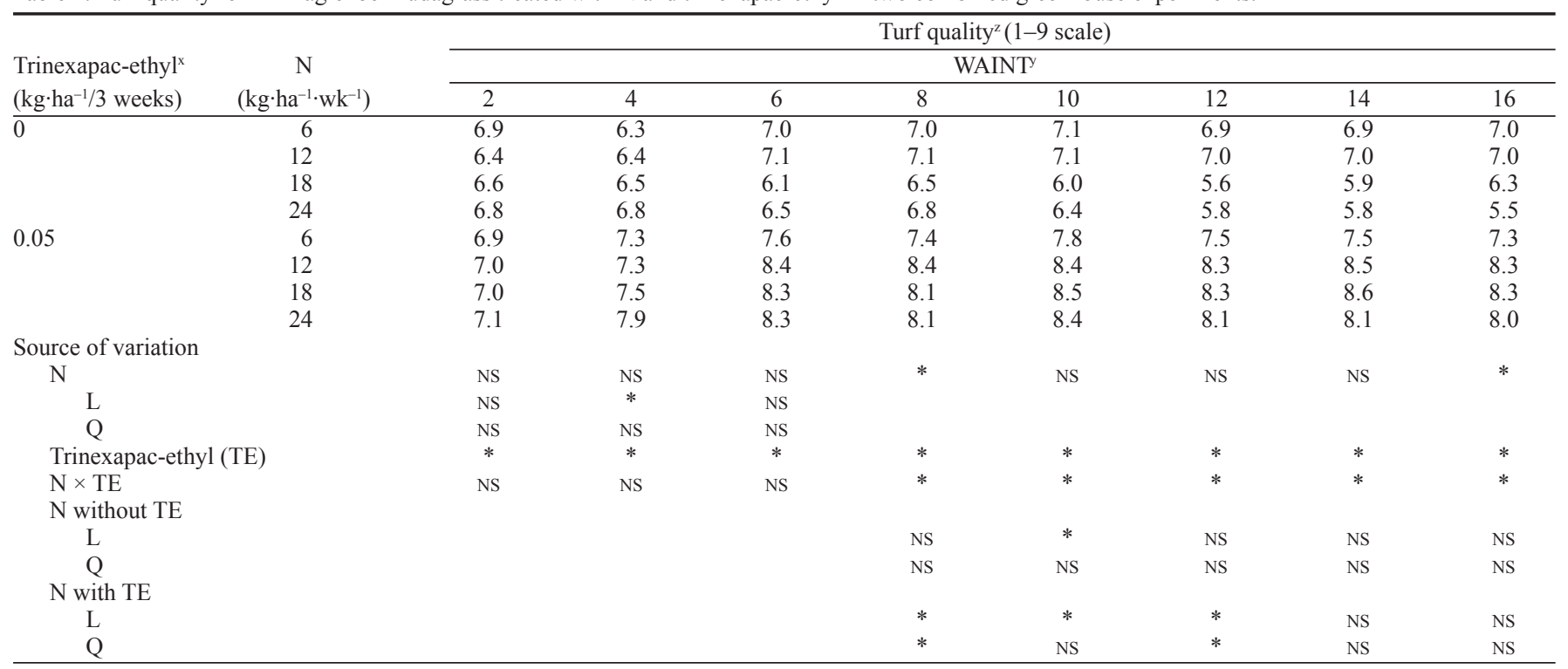

${ }^{2}$ Turf quality was rated 1 to 9 where $1=$ dead or dormant and $9=$ dark green, uniform turf.

${ }^{\mathrm{y}} \mathrm{WAINT}=$ weeks after initial $\mathrm{N}$ treatment.

${ }^{x}$ Initial trinexapac-ethyl treatment was $9 \mathrm{~d}$ after initial $\mathrm{N}$ treatments.

NS,"Nonsignificant or significant at the 0.05 probability level. $\mathrm{L}=$ linear, $\mathrm{Q}=$ quadratic. 
sampling dates (Table 3). Bermudagrass treated with TE had leaf $\mathrm{N}$ concentrations reduced $6 \%$

on week 3, one WAIT (Fig. 1). Leaf N concentrations were lower in TE treated turf, ranging

Table 2. Total clipping yield, dry root mass, and root length after 16 weeks for 'TifEagle' bermudagrass treated with $\mathrm{N}$ and trinexapac-ethyl in two combined greenhouse experiments.

\begin{tabular}{|c|c|c|c|c|}
\hline $\begin{array}{l}\text { Trinexapac-ethyl } \\
\left(\mathrm{kg} \cdot \mathrm{ha}^{-1} / 3 \text { weeks }\right)\end{array}$ & $\begin{array}{c}\mathrm{N} \\
\left(\mathrm{kg} \cdot \mathrm{ha}^{-1} \cdot \mathrm{wk}^{-1}\right)\end{array}$ & $\begin{array}{c}\text { Total } \\
\text { clippings } \\
\left(\mathrm{g} \cdot \mathrm{m}^{-2}\right)\end{array}$ & $\begin{array}{c}\text { Root } \\
\text { mass }^{\mathrm{y}} \\
\left(\mathrm{g} \cdot \mathrm{m}^{-2}\right)\end{array}$ & $\begin{array}{c}\text { Root } \\
\text { length } \\
(\mathrm{cm})\end{array}$ \\
\hline \multirow[t]{4}{*}{0} & 6 & 12.2 & 26.9 & 22.9 \\
\hline & 12 & 26.9 & 23.5 & 21.0 \\
\hline & 18 & 32.7 & 18.8 & 16.1 \\
\hline & 24 & 37.6 & 19.9 & 16.8 \\
\hline \multirow[t]{4}{*}{0.05} & 6 & 5.8 & 40.4 & 22.4 \\
\hline & 12 & 10.4 & 28.8 & 26.7 \\
\hline & 18 & 14.3 & 28.9 & 22.2 \\
\hline & 24 & 14.7 & 28.8 & 23.4 \\
\hline \multicolumn{5}{|l|}{ Source of variation } \\
\hline $\mathrm{N}$ & & $*$ & $*$ & $*$ \\
\hline $\mathrm{L}$ & & & $*$ & $*$ \\
\hline Q & & & $*$ & NS \\
\hline Trinexapac-eth & & $*$ & $*$ & $*$ \\
\hline $\mathrm{N} \times \mathrm{TE}$ & & $*$ & NS & NS \\
\hline \multicolumn{5}{|l|}{$\mathrm{N}$ without TE } \\
\hline Linear & & $*$ & & \\
\hline Q & & $*$ & & \\
\hline \multicolumn{5}{|l|}{$\mathrm{N}$ with $\mathrm{TE}$} \\
\hline L & & $*$ & & \\
\hline $\mathrm{Q}$ & & $*$ & & \\
\hline
\end{tabular}

zTotal of 16 dry weight clipping yields harvested from weekly samples.

${ }^{y}$ Root mass and root length samples were harvested 16 weeks after initial $\mathrm{N}$ treatment from entire containers.

NS,"Nonsignificant or significant at the 0.05 probability level. $\mathrm{L}=$ linear, $\mathrm{Q}=$ quadratic. from $2 \%$ to $6 \%$ reductions, through week 12 . $\mathrm{N}$ concentrations in leaves and belowground tissues ascended linearly with increased $\mathrm{N}$ rate (Table 3). Root $\mathrm{N}$ concentrations increased in TE treated turf by $8 \%$ when fertilized at 12 $\mathrm{kg} \mathrm{N} / \mathrm{ha} /$ week and $11 \%$ when fertilized at 18 and $24 \mathrm{~kg} \mathrm{~N} / \mathrm{ha} /$ week compared to respective $\mathrm{N}$ rates of non-TE treated. Higher $\mathrm{N}$ rates increased $\mathrm{P}$ concentrated in leaf tissue and reduced $\mathrm{P}$ concentrations in roots, stolons, and rhizomes. Similarly, increased N rate reduced root, stolon, and rhizome $\mathrm{K}$ concentrations. 'TifEagle' bermudagrass treated with TE had similar root $\mathrm{K}$ concentrations across $\mathrm{N}$ rates and approximately $25 \%$ greater $\mathrm{K}$ concentrations than nontreated turf after 16 weeks.

TE-treated bermudagrass had higher leaf $\mathrm{Ca}$ and $\mathrm{Mg}$ concentrations, increasing by as much as $27 \%$ and $11 \%$, respectively, on four sampling dates (Fig. 1). Bermudagrass treated with TE had similar primary and secondary nutrient concentrations in stolons and rhizomes compared to non-TE treated turf. $\mathrm{N} \times \mathrm{TE}$ interaction was detected for root $\mathrm{Mg}$ concentrations, as TE masked concentration reductions caused by increased $\mathrm{N}$ rate (Table 3). Increased $\mathrm{N}$ rate caused reductions in stolon and rhizome $\mathrm{S}$ concentrations but TE treated turf was similar to non-TE treated.

Table 3. Leaf, root, and stolon-rhizome nutrient concentrations after 16 weeks for 'TifEagle' bermudagrass treated with $\mathrm{N}$ and trinexapac-ethyl in two combined greenhouse experiments.

\begin{tabular}{|c|c|c|c|c|c|c|c|c|c|c|c|}
\hline \multirow{3}{*}{$\begin{array}{l}\text { Trinexapac-ethyl }{ }^{\mathrm{z}} \\
\left(\mathrm{kg} \cdot \mathrm{ha}^{-1} / 3 \text { weeks }\right)\end{array}$} & \multirow{3}{*}{$\begin{array}{c}\mathrm{N} \\
\left(\mathrm{kg} \cdot \mathrm{ha}^{-1} \cdot \mathrm{wk}^{-1}\right)\end{array}$} & \multicolumn{10}{|c|}{ Nutrient concn } \\
\hline & & $\mathrm{N}$ & $\mathrm{P}$ & $\mathrm{K}$ & $\mathrm{Ca}$ & $\mathrm{Mg}$ & $\mathrm{S}$ & $\mathrm{Zn}$ & $\mathrm{Cu}$ & $\mathrm{Mn}$ & $\mathrm{Fe}$ \\
\hline & & \multicolumn{6}{|c|}{$(\%)$} & \multicolumn{4}{|c|}{$\left(\mathrm{mg} \cdot \mathrm{kg}^{-1}\right)$} \\
\hline \multicolumn{12}{|l|}{ Leaves } \\
\hline & 6 & 4.38 & 0.44 & 1.59 & 0.37 & 0.24 & 0.38 & 61 & 21 & 71 & 205 \\
\hline & 12 & 5.22 & 0.47 & 1.62 & 0.35 & 0.24 & 0.38 & 66 & 21 & 102 & 189 \\
\hline & 18 & 5.58 & 0.48 & 1.63 & 0.39 & 0.24 & 0.38 & 73 & 22 & 125 & 189 \\
\hline & 24 & 5.72 & 0.49 & 1.65 & 0.36 & 0.24 & 0.37 & 70 & 21 & 135 & 174 \\
\hline $\mathrm{L}$ & & $*$ & * & $*$ & $*$ & NS & NS & $*$ & NS & $*$ & * \\
\hline Q & & NS & NS & NS & $*$ & NS & NS & * & NS & NS & $*$ \\
\hline \multicolumn{12}{|l|}{ Roots } \\
\hline \multirow[t]{4}{*}{0} & 6 & 1.27 & 0.11 & 0.51 & 0.16 & 0.08 & 0.15 & 50 & 29 & 15 & 206 \\
\hline & 12 & 1.29 & 0.08 & 0.33 & 0.14 & 0.07 & 0.12 & 54 & 30 & 40 & 227 \\
\hline & 18 & 1.63 & 0.08 & 0.26 & 0.12 & 0.06 & 0.13 & 58 & 41 & 46 & 308 \\
\hline & 24 & 1.64 & 0.09 & 0.28 & 0.15 & 0.06 & 0.13 & 75 & 50 & 68 & 551 \\
\hline \multirow[t]{4}{*}{0.05} & 6 & 1.15 & 0.11 & 0.48 & 0.12 & 0.07 & 0.13 & 40 & 23 & 19 & 167 \\
\hline & 12 & 1.40 & 0.09 & 0.43 & 0.12 & 0.07 & 0.13 & 47 & 22 & 30 & 284 \\
\hline & 18 & 1.82 & 0.11 & 0.45 & 0.14 & 0.08 & 0.15 & 65 & 33 & 45 & 361 \\
\hline & 24 & 1.83 & 0.10 & 0.42 & 0.12 & 0.07 & 0.12 & 56 & 26 & 58 & 280 \\
\hline \multicolumn{12}{|l|}{ Source of variation } \\
\hline $\mathrm{N}$ & & $*$ & $*$ & $*$ & NS & NS & NS & * & NS & $*$ & $*$ \\
\hline $\mathrm{L}$ & & $*$ & NS & & NS & & NS & * & $*$ & * & $*$ \\
\hline Q & & NS & $*$ & & NS & & NS & NS & NS & NS & NS \\
\hline Trinexapac-eth & TE) & NS & NS & $*$ & NS & NS & NS & NS & $*$ & NS & NS \\
\hline $\mathrm{N} \times \mathrm{TE}$ & & NS & NS & $*$ & NS & $*$ & NS & NS & NS & NS & NS \\
\hline \multicolumn{12}{|l|}{$\mathrm{N}$ without $\mathrm{TE}$} \\
\hline $\mathrm{L}$ & & & & $*$ & & $*$ & & & & & \\
\hline Q & & & & $*$ & & NS & & & & & \\
\hline \multicolumn{12}{|l|}{$\mathrm{N}$ with TE } \\
\hline $\mathrm{L}$ & & & & NS & & NS & & & & & \\
\hline $\mathrm{Q}$ & & & & NS & & NS & & & & & \\
\hline \multicolumn{12}{|c|}{ Stolons and rhizomes } \\
\hline & 6 & 1.27 & 0.15 & 0.47 & 0.20 & 0.47 & 0.09 & 95 & 20 & 44 & 602 \\
\hline & 12 & 1.57 & 0.12 & 0.37 & 0.18 & 0.37 & 0.08 & 97 & 20 & 59 & 692 \\
\hline & 18 & 1.93 & 0.11 & 0.32 & 0.15 & 0.32 & 0.07 & 100 & 23 & 77 & 820 \\
\hline & 24 & 2.13 & 0.12 & 0.31 & 0.18 & 0.31 & 0.08 & 110 & 25 & 104 & 990 \\
\hline $\mathrm{L}$ & & $*$ & * & $*$ & NS & * & NS & NS & $*$ & $*$ & * \\
\hline $\mathrm{Q}$ & & NS & $*$ & NS & NS & NS & $*$ & NS & NS & $*$ & NS \\
\hline 0 & & 1.7 & 0.1 & 0.4 & 0.2 & 0.1 & 0.1 & 108 & 24 & 88 & 821 \\
\hline 0.05 & & 1.8 & 0.1 & 0.4 & 0.2 & 0.1 & 0.1 & $93^{*}$ & $20^{*}$ & $75^{*}$ & 731 \\
\hline
\end{tabular}

${ }^{\text {ZInitial trinexapac-ethyl treatment was week } 2 .}$

NS, ${ }^{*}$ Nonsignificant or significant at the 0.05 probability level. $\mathrm{L}=$ linear, $\mathrm{Q}=$ quadratic. 

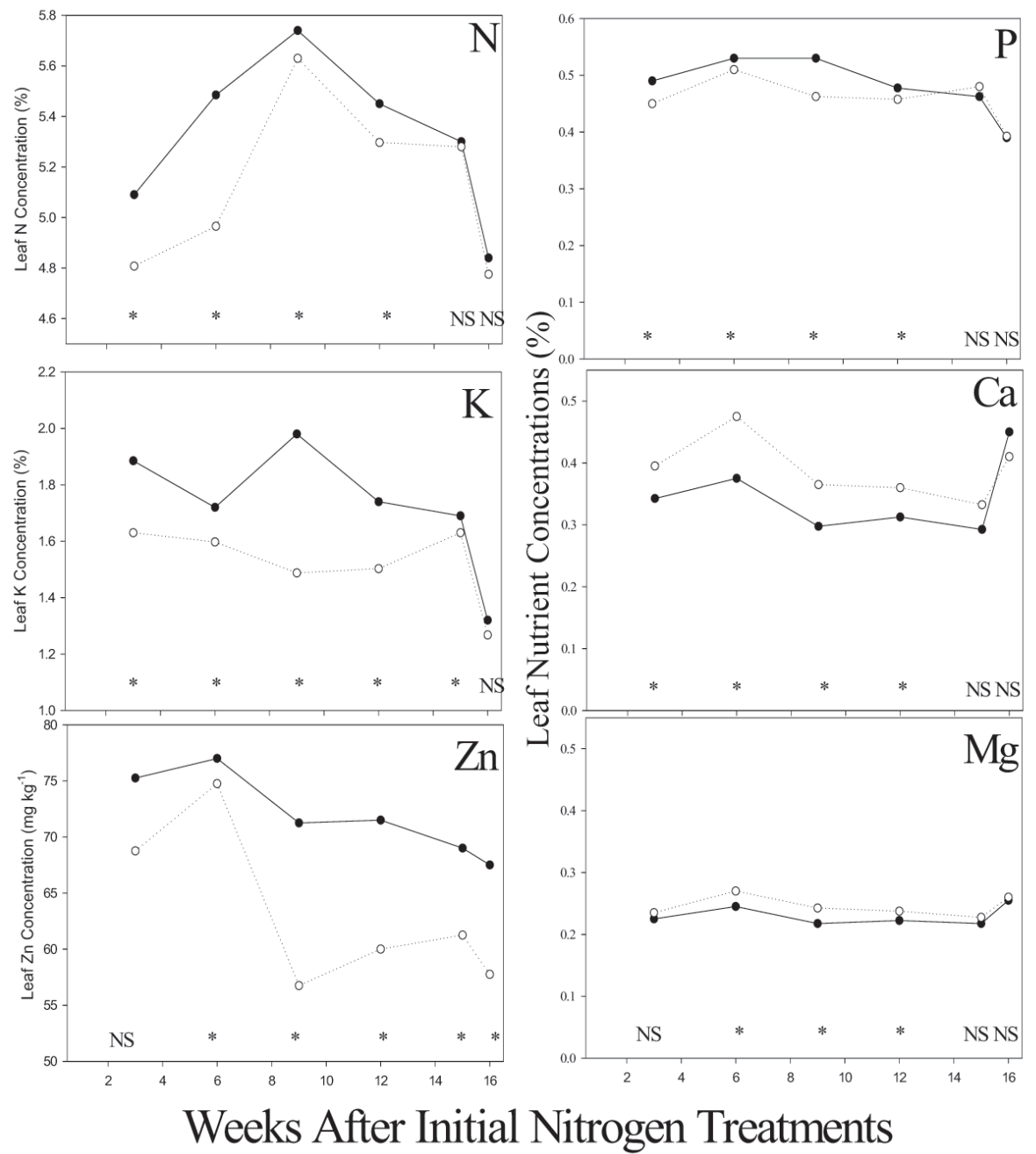

Fig. 1. Leaf nutrient concentrations for 'TifEagle' bermudagrass treated with trinexapac-ethyl at 0 and $0.05 \mathrm{~kg} \cdot \mathrm{ha}^{-1}$ per 3 weeks beginning $9 \mathrm{~d}$ after initial nitrogen treatments in two combined greenhouse experiments. ( $\longrightarrow$ ) Nontreated, (------) treated
For micronutrients, higher $\mathrm{N}$ inputs increased bermudagrass leaf $\mathrm{Zn}$ and $\mathrm{Mn}$ concentrations (Table 3). Leaf Zn concentrations were reduced in TE treated turf on five sampling dates from the nontreated (Fig. 1). Other leaf micronutrients concentrations were similar to non-TE treated turf (data not shown). Increased $\mathrm{N}$ rate resulted in higher micronutrient concentrations in roots, stolons, and rhizomes. Bermudagrass treated with TE had $\mathrm{Zn}, \mathrm{Cu}$, and $\mathrm{Mn}$ concentrations reduced in stolons and rhizomes by about $15 \%$ and root $\mathrm{Cu}$ concentrations reduced $30 \%$ from non-TE treated turf. Increased $\mathrm{N}$ rate linearly increased Fe concentrations of belowground tissues and reduced $\mathrm{N}$ concentrated in leaves (Table 3 ). 'TifEagle' bermudagrass treated with TE had similar $\mathrm{Fe}$ concentrations to the nontreated (data not shown).

Nutrient recovery. An $\mathrm{N} \times \mathrm{TE}$ interaction was not detected for final leaf nutrient recovery in Study 1.After 16 weeks in Study 1, increased $\mathrm{N}$ rate generally increased clipping nutrient recovery while TE-treated turfaveraged $30 \%$ to $75 \%$ reduced nutrient recoveries from non-TE treated (Table 4). From six sampling dates in Study 2, N $\times$ TE interactions were detected for total $\mathrm{N}, \mathrm{P}, \mathrm{K}, \mathrm{Mg}, \mathrm{S}, \mathrm{Zn}, \mathrm{Cu}$, and $\mathrm{Mn}$ recovered in clippings. Increased nutrient recovery occurred with increased $\mathrm{N}$ rates; however, total nutrients recovered were reduced $69 \%$ to $79 \%$ in the presence of TE. Bermudagrass treated with $\mathrm{TE}$, regardless of $\mathrm{N}$ input, had clipping $\mathrm{N}$ recovery less than turf receiving $12 \mathrm{~kg} \mathrm{~N} /$ ha/week without TE and had similar clipping recoveries to turf treated with $6 \mathrm{~kg} \mathrm{~N} / \mathrm{ha} /$ week without TE for all other mineral nutrients.

'TifEagle' bermudagrass treated with TE had $50 \%$ greater $\mathrm{N}$ recovered in roots after 16 weeks compared to non-TE treated turf (Table 5). Bermudagrass treated with $24 \mathrm{~kg}$

Table 4. Clipping nutrient recovery for 'TifEagle' bermudagrass treated with nitrogen and trinexapac-ethyl in greenhouse experiments.

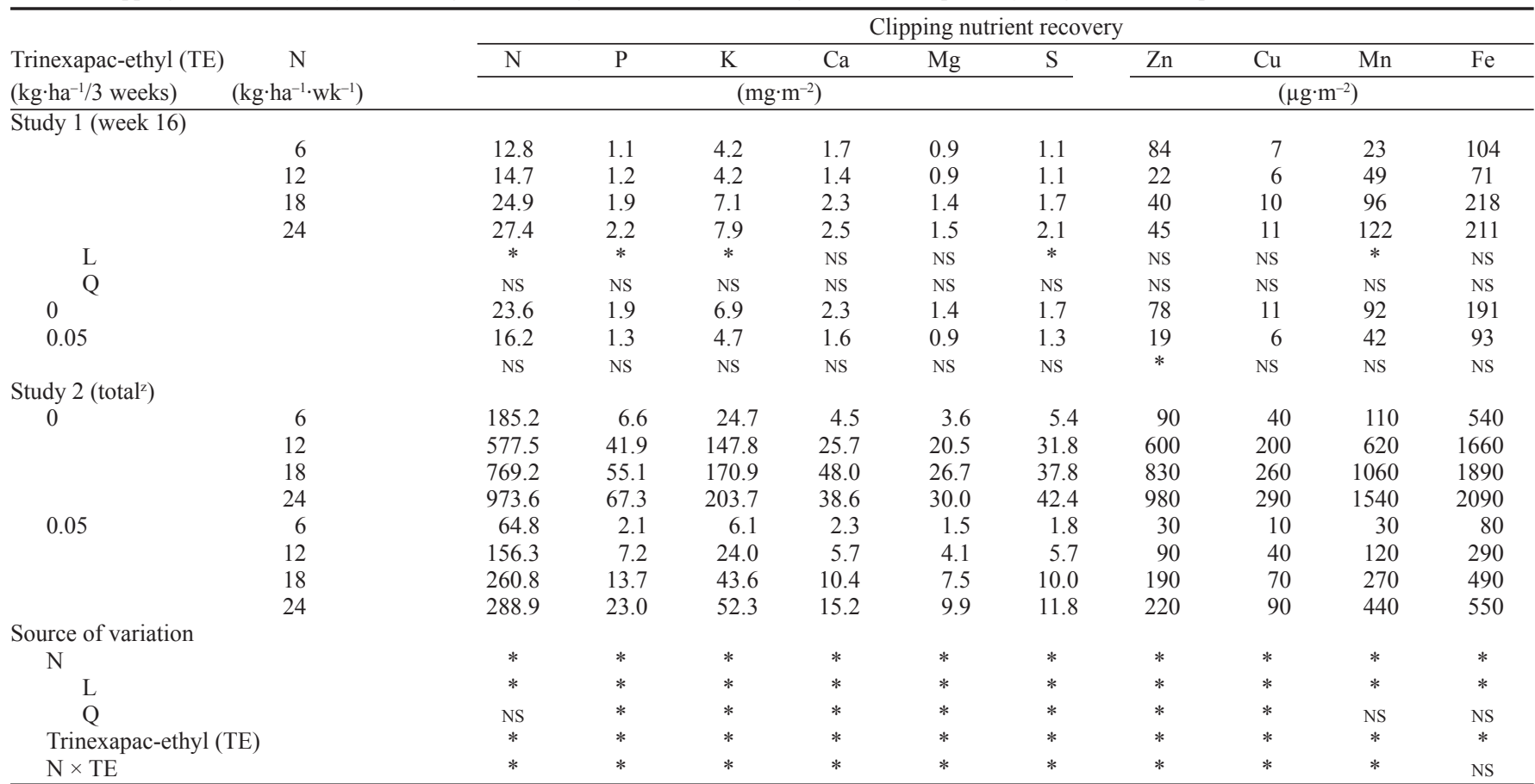

${ }^{2}$ Tissue tests in Study 2 were conducted 3, 6, 9, 12, 15, and 16 weeks after initial $\mathrm{N}$ treatment.

NS,"Nonsignificant or significant at the 0.05 probability level. $\mathrm{L}=$ linear, $\mathrm{Q}=$ quadratic. 
Table 5. Root, stolon, and rhizome nutrient retention after 16 weeks for 'TifEagle' bermudagrass treated with nitrogen and trinexapac-ethyl in two combined greenhouse experiments.

\begin{tabular}{|c|c|c|c|c|c|c|c|c|c|c|c|}
\hline \multirow{2}{*}{$\begin{array}{l}\text { Trinexapac-ethyl } \\
\left(\mathrm{kg} \cdot \mathrm{ha}^{-1} / 3 \text { weeks }\right)\end{array}$} & \multirow{2}{*}{$\begin{array}{c}\mathrm{N} \\
\left(\mathrm{kg} \cdot \mathrm{ha}^{-1} \cdot \mathrm{wk}^{-1}\right)\end{array}$} & \multicolumn{10}{|c|}{ Nutrient retention $\left(\mathrm{mg} \cdot \mathrm{m}^{-2}\right)$} \\
\hline & & $\mathrm{N}$ & $\mathrm{P}$ & $\mathrm{K}$ & $\mathrm{Ca}$ & $\mathrm{Mg}$ & $\mathrm{S}$ & $\mathrm{Zn}$ & $\mathrm{Cu}$ & $\mathrm{Mn}$ & $\mathrm{Fe}$ \\
\hline \multicolumn{12}{|l|}{ Roots } \\
\hline & 6 & 395.5 & 34.8 & 154.2 & 43.6 & 24.5 & 44.0 & 1.4 & 0.9 & 0.6 & 15.1 \\
\hline & 12 & 350.1 & 22.5 & 101.5 & 33.3 & 18.6 & 32.8 & 1.2 & 0.6 & 0.7 & 5.5 \\
\hline & 24 & 427.7 & 22.9 & 86.8 & 31.9 & 16.6 & 30.4 & 1.5 & 0.9 & 1.5 & 8.9 \\
\hline $\mathrm{L}$ & & NS & * & $*$ & * & * & * & NS & NS & $*$ & NS \\
\hline Q & & NS & $*$ & * & NS & * & * & $*$ & * & NS & NS \\
\hline & & * & $*$ & $*$ & * & $*$ & $*$ & $*$ & NS & $*$ & NS \\
\hline \multicolumn{12}{|c|}{ Stolons and rhizomes } \\
\hline & 6 & 9.0 & 1.0 & 3.3 & 1.2 & 0.6 & 1.1 & 39.1 & 9.1 & 45.45 & 266.9 \\
\hline & 12 & 11.8 & 0.9 & 3.0 & 1.2 & 0.6 & 1.0 & 42.9 & 9.7 & 54.05 & 297.3 \\
\hline & 18 & 13.9 & 0.7 & 2.0 & 1.1 & 0.5 & 0.9 & 43.7 & 10.9 & 60.90 & 364.8 \\
\hline & 24 & 15.9 & 0.9 & 2.4 & 1.2 & 0.5 & 1.0 & 47.7 & 11.7 & 79.25 & 448.9 \\
\hline $\mathrm{L}$ & & $*$ & NS & $*$ & NS & $*$ & NS & $*$ & $*$ & * & * \\
\hline
\end{tabular}

Initial trinexapac-ethyl treatment was week 2 .

NS,"Nonsignificant or significant at the 0.05 probability level. $\mathrm{L}=$ linear, $\mathrm{Q}=$ quadratic.

Table 6. Soil nitrate-nitrogen $\left(\mathrm{NO}_{3}-\mathrm{N}\right)$ concentration after 16 weeks for 'TifEagle' bermudagrass treated with nitrogen in two combined greenhouse experiments.

\begin{tabular}{lc}
\hline $\begin{array}{l}\mathrm{N}^{\mathrm{z}} \\
\left(\mathrm{kg} \cdot \mathrm{ha}^{-1} \cdot \mathrm{wk}^{-1}\right)\end{array}$ & $\begin{array}{c}\text { Soil NO} \\
\left(\mathrm{mg} \cdot \mathrm{kg}^{-1}\right)\end{array}$ \\
\hline 6 & 2.1 \\
12 & 2.5 \\
18 & 4.7 \\
24 & 6.8 \\
$\mathrm{~L}$ & $*$ \\
$\mathrm{Q}$ & $\mathrm{NS}$ \\
\hline
\end{tabular}

${ }^{2}$ Nitrogen source was $34 \mathrm{~N}-0 \mathrm{P}-0 \mathrm{~K}$ ammonium nitrate.

NS,"Nonsignificant or significant at the 0.05 probability level. $\mathrm{L}=$ linear, $\mathrm{Q}=$ quadratic.

$\mathrm{N} /$ ha/week had $22 \%$ higher root $\mathrm{N}$ recovery compared to $12 \mathrm{~kg} \mathrm{~N} /$ ha/week fertilized turf. Bermudagrass treated with TE had $25 \%$ to $105 \%$ greater nutrients recovered in roots compared to non-TE treated turf. Stolon and rhizome $\mathrm{N}$ and $\mathrm{Mn}$ retention increased with $\mathrm{N}$ rate after 16 weeks. 'TifEagle' bermudagrass treated with TE had similar nutrient retention in stolons and rhizomes as non-TE treated turf (data not shown). Soil $\mathrm{NO}_{3}-\mathrm{N}$ concentrations of TE treated turf were similar to nontreated (data not shown) but concentrations linearly increased with $\mathrm{N}$ rates (Table 6).

Chlorophyll concentrations. Nitrogen $\times \mathrm{TE}$ interactions were not detected for chlorophyll concentrations (CC). A quadratic relationship was detected for $\mathrm{N}$ rate and CC 8 WAINT in Study 2 (Table 7). Increased $\mathrm{N}$ rate from 6 to $18 \mathrm{~kg} \mathrm{~N} / \mathrm{ha} /$ week enhanced CC but declined with $24 \mathrm{~kg} \mathrm{~N} / \mathrm{ha} /$ week. Turf treated with TE had about $65 \%$ higher total CC 8 WAIT in Study 1. 'TifEagle' treated with TE had about $40 \%$ higher CC 12 WAIT in Study 2. CC was similar among treatments after 16 weeks (data not shown).

\section{Discussion}

'TifEagle' and other dwarf bermudagrasses require ample $\mathrm{N}$ fertility to promote turfgrass color and quality. However, rooting of these grasses may decline as persistent shoot growth competes for root reserves. Reducing uneven shoot growth with applications of TE may promote surface uniformity and balance nutrient partitioning within the plant. Redirected plant energy away from new leaf growth may therefore provide more favorable growing conditions for dwarf bermudagrass root systems.

Increased $\mathrm{N}$ rates resulted in higher $\mathrm{P}$ concentrations in leaf tissue and reduced $\mathrm{P}$ concentrations in roots and rhizomes. Conversely, leaf $\mathrm{N}, \mathrm{P}$, and $\mathrm{K}$ concentrations were reduced 1 week after initial TE application, while increased concentrations were observed in roots after 16 weeks. Results are consistent with reports of TE reducing Nallocation to 'Tifway' bermudagrass leaf tissue while increasing $\mathrm{N}$ retention in roots (Fagerness et al., 2004). More importantly, these results exemplify an altered source and sink relationship under shoot growth inhibition which likely is attributed to root mass and root length enhancements using TE.

'TifEagle' bermudagrass, under shoot growth inhibition, reallocated $\mathrm{N}$ and all other nutrients away from leaf tissue. Greater nutrients stored in belowground tissues could therefore be allocated to prevent leaf nutrient

Table 7. Chlorophyll concentrations for 'TifEagle' bermudagrass treated with $\mathrm{N}$ and trinexapac-ethyl in greenhouse experiments.

\begin{tabular}{|c|c|c|c|}
\hline \multirow{3}{*}{$\begin{array}{l}\text { Trinexapac-ethyl }{ }^{y} \\
\left(\mathrm{~kg} \cdot \mathrm{ha}^{-1} / 3 \text { weeks }\right)\end{array}$} & \multirow{3}{*}{$\begin{array}{c}\mathrm{N} \\
\left(\mathrm{kg} \cdot \mathrm{ha}^{-1} \cdot \mathrm{wk}^{-1}\right)\end{array}$} & \multicolumn{2}{|c|}{ Total chlorophyll concn $\left(\mathrm{mg} \cdot \mathrm{g}^{-1}\right)$} \\
\hline & & \multicolumn{2}{|c|}{ WAINT $^{\mathrm{z}}$} \\
\hline & & 8 & 12 \\
\hline \multicolumn{4}{|l|}{ Study 1} \\
\hline 0 & & 6.2 & \\
\hline 0.05 & & $10.3^{*}$ & \\
\hline \multicolumn{4}{|l|}{ Study 2} \\
\hline & 6 & 10.1 & 8.1 \\
\hline & 12 & 13.4 & 8.3 \\
\hline & 18 & 11.3 & 8.0 \\
\hline & 24 & 10.0 & 8.0 \\
\hline $\mathrm{L}$ & & $*$ & NS \\
\hline Q & & * & NS \\
\hline 0 & & 10.4 & 7.0 \\
\hline 0.05 & & 11.4 & 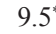 \\
\hline
\end{tabular}

${ }^{2}$ WAINT $=$ weeks after initial $\mathrm{N}$ treatment.

${ }^{y}$ Initial trinexapac-ethyl treatment was week 2.

NS,"Nonsignificant or significant at the 0.05 probability level. $\mathrm{L}=$ linear, $\mathrm{Q}=$ quadratic.

deficiencies. Since bermudagrass putting greens are heavily fertilized, improved nutrient use efficiency could reduce high fertility requirements of these grasses. Reductions in leaf micronutrients concentrations in TE treated turf were not consistent with primary and secondary nutrient concentrations. However, it could be inferred that requirements for micronutrients may be reduced under long-term leaf growth inhibition.

Inhibiting shoot growth with TE reduced nutrients removed through clippings and caused higher chlorophyll concentrations per unit leaf area. Consequently, color enhancements resulted to where fertilizations may be unnecessary to promote turf quality. 'TifEagle' bermudagrass may necessitate between 6 to $12 \mathrm{~kg} \mathrm{~N} /$ ha/week to promote turf quality and color enhancements when using TE. Moreover, bermudagrass quality enhancements from TE applications were most substantial when fertilized greater than $6 \mathrm{~kg} \mathrm{~N} / \mathrm{ha}$ and may only be significant when adequate $\mathrm{N}$ is available for growth.

The absence of a significant Nx TE interaction suggests TE applications helped mitigate but did not completely mask the influence of $\mathrm{N}$ fertility on 'TifEagle' bermudagrass
HortScience Vol. 41(2) ApriL 2005 
root growth. Inhibited leaf growth apparently reduced shoot competition for root reserves, thus providing more favorable conditions for growth. Nitrogen distribution away from leaves may have stimulated 'TifEagle' bermudagrass root growth from increased nutrient and energy partitioning to roots. 'TifEagle' bermudagrass shoot growth, under regulation by TE, may no longer be as competitive with roots for nutrients and plant reserves. Overall, routine applications of TE may encourage bermudagrass root growth and enhance nutrient use efficiency, thus making an effective tool for long-term dwarf bermudagrass culture.

\section{Literature Cited}

Fagerness, M.J. and F.H. Yelverton. 2000. Tissue production and quality of Tifway bermudagrass as affected by seasonal application patterns of trinexapac-ethyl. Crop Sci. 40:493-497.

Fagerness, M.J. and F.H Yelverton. 2001. Plant growth regulator and mowing height effects on seasonal root growth of Penncross creeping bentgrass. Crop Sci. 41:1901-1905.

Fagerness, M.J., D.C. Bowman, F.H. Yelverton, and T.W. Rufty. 2004. Nitrogen use in Tifway bermudagrass, as influenced by trinexapac-ethyl. Crop Sci. 44:595-599.

Goss, R.M., J.H. Baird, S.L. Kelm, and R.M. Calhoun. 2002. Trinexapac-ethyl and nitrogen effects on creeping bentgrass grown under reduced light conditions. Crop Sci. 42:472-479.

Hanna, W.W. and J.L. Elsner. 1999. Registration of TifEagle bermudagrass. Crop Sci. 39:1258.

Johnson, B.J. 1997. Growth of 'Tifway' bermudagrass following application of nitrogen and iron with trinexapac-ethyl. HortScience 32(2):241-242.

Liu, X. and B. Huang. 2002. Mowing effects on root production, growth, and mortality of creeping bentgrass. Crop Sci. 42:1241-1251.

McCarty, L.B. and G.L. Miller. 2002. Managing bermudagrass turf: Selection, construction, cultural practices and pest management strategies, p. 7, 36. Sleeping Bear Press, Chelsea, Mich.
Moran, R. and D. Porath. 1980. Chlorophyll determination in intact tissues using N,N-dimethylforamide. Plant Physiol. 65:478-479.

Qian, Y.L. and M.C. Engelke. 1999. Influence of trinexapac-ethyl on 'Diamond' zoysiagrass in a shade environment. Crop Sci. 39:202-208.

Rademacher, W. 2000. Growth retardants: Effects on gibberellin biosynthesis and other metabolic pathways. Ann. Rev. Plant Physiol. Plant Mol. Biol. 51:501-531.

Richardson, M.D. 2002. Turf quality and freezing tolerance of Tifway bermudagrass as affected by late-season nitrogen and trinexapac-ethyl. Crop Sci. 42:162-166.

SAS Institute. 1999. The SAS system for Windows. v. 8.2. SAS Inst., Cary, N.C.

U.S. Golf Association Green Section Staff. 1993. USGA recommendations for a method of putting green construction. The 1993 Revision. USGA Green Sect. Record 31(2):1-3.

White, R.H. 1998. 1998 Semi-annual research progress report of performance and management of new dwarf bermudagrasses. Texas A \& M Univ. p. 12. 